\title{
Superconformal Compactifications in Weighted Projective Space
}

\author{
B. R. Greene* \\ Lyman Laboratory of Physics, Harvard University, Cambridge, MA 02138, USA
}

\begin{abstract}
We discuss some aspects of string vacua constructed from orbifolded nonminimal Landau-Ginzburg theories which correspond to Calabi-Yau manifolds in weighted projective space. In contrast to previous expectations, we find that these theories allow for the construction of numerous stable $(2,0)$ Calabi-Yau vacua (most of which are not simply deformations of an underlying $(2,2)$ theory) thus indicating that this phenomenologically promising sector of the space of classical vacua is quite robust. We briefly discuss methods for extracting the phenomenology of these models and show, for example, that the full renormalizable superpotential of our $S U(5)$ theories is not corrected by world sheet instantons and is thus given exactly by its tree-level value.
\end{abstract}

\section{Introduction}

The initial attempts at constructing four dimensional string theories stressed a geometric interpretation $[1,2]$ for the extra degrees of freedom required by the demands of an underlying superconformal symmetry. It was found that six dimensions should be compactified on a complex threefold with vanishing Ricci tensor. This tether to geometry was gradually slackened by a number of groups [3-7] whose work emphasized that so long as the extra degrees of freedom meet the demands of conformal symmetry, there is no need for them to admit a geometric interpretation. In particular, any unitary $c=9, N=2$ superconformal theory may be chosen for the internal degrees of freedom for a four dimensional superstring theory. A general method of converting such a superstring theory into a heterotic string theory was given in [7]. This philosophy was pursued further in [8], with the emphasis on using the simplest nontrivial $N=2$ theories: the minimal models. The surprising observation of [8] is the unexpected reemergence of geometry: numerous correspondences between detailed properties of the minimal model

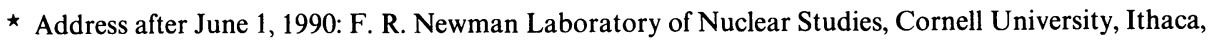
NY 14853, USA 
theories and certain Calabi-Yau compactifications led to their conjectured equivalence [8]. This conjecture was subsequently proved in [9-10]. Even with our understanding of this connection, it is still rather remarkable that the complicated field theory of Calabi-Yau sigma models (one can not even write down the tree level action in closed form) can be described by some of the simplest conformal theories. Thus, among other things, this correspondence serves to illustrate an important and a priori unsuspected capability of conformal theories to offer vastly simplified descriptions of complicated geometrical constructions. On the other hand, the geometrical viewpoint provides a very useful and important organizing principle for the algebraic conformal field theory constructions. For example, the massless spectra, couplings and discrete symmetries of the latter may be identified with the geometric cohomology classes, cohomological ring structure and holomorphic automorphisms. In fact, many of these geometric notions find application even in instances for which there is (at present) no obvious geometric counterpart to the algebraic construction. Each perspective, therefore, serves to enhance the other. The geometric approach provides an organizing framework for the detailed characteristics of these theories; the algebraic approach makes it clear that the prima facie complexity of the geometrical construction is, from a pragmatic viewpoint, largely an illusion. It is likely that the interplay between geometry and conformal field theory will play a key role in helping to unravel many of the mysteries of string theory.

The purpose of this paper is to present some new geometrical constructions of string vacua which, among other things, show that a number of the pleasing properties of $(2,2)$ theories persist in the more general $(2,0)$ vacua. We begin by discussing theories with $(2,2)$ world sheet supersymmetry that are generalizations of the Landau-Ginzburg theories invoked in [9-10]. Geometrically, these vacua correspond to new constructions of Calabi-Yau manifolds in weighted projective space. From the Landau-Ginzburg point of view, they correspond to higher modality catastrophic superpotentials. These constructions in weighted projective space have the simplicity of the well studied complete intersections in ordinary projective space but also naturally incorporate the quotient singularities which are inherent to the toroidal orbifold approach. Thus, these examples, which can have negative as well as positive Euler numbers, serve as a unifying bridge between these two classes of geometric string vacua.

After describing some of these theories, we make use of them to construct $(2,0)$ string vacua. It has long been known that phenomenology favours $(2,0)$ theories over $(2,2)$ theories. Most work on $(2,0)$ theories with a geometrical basis has been based on modifying an underlying $(2,2)$ theory in one of two ways: either one adds Wilson loops (or, more appropriately "gauge twists") [11-13] or one holomorphically deforms the $(2,2)$ theory by turning on a Planck scale vacuum expectation value for a massless mode which has an exactly flat superpotential [14-15]. Our interest focuses not on these constructions, but rather on the potentially rich and largely unexplored sector of the space of $(2,0)$ vacua which are not simply modifications or deformations of underlying $(2,2)$ vacua. The reason for the paucity of work on this phenomenologically favoured class of solutions stemmed initially from the results of [16]. In [16] it was argued that world sheet instantons will generically destabilize $(2,0)$ Calabi-Yau vacua. Subsequently, 
though, it was shown in [17] that a simple quasi-topological condition on a would-be $(2,0)$ vacuum ensures that this destabilization is evaded. Constructing examples which realize this mechanism for avoiding destabilization, however, has proven difficult. In [17] only one example was found and it may in fact be that this example is, in reality, simply a deformation of an underlying $(2,2)$ theory. We show here that by going beyond complete intersection Calabi-Yau manifolds to those embedded in weighted projective space we are able to construct numerous stable $(2,0)$ string vacua. These examples are of significance for three reasons. First, since they are not connected to any underlying $(2,2)$ theory, they conclusively show that there are a wealth of interesting vacua which cannot be reached by the procedure of holomorphic deformation advocated in [14] and [15]. This is a complication in the structure of the moduli space of geometric string vacua which any classification program must ultimately address. Second, from the point of view of model building, these vacua are quite promising. Whereas $(2,2)$ theories give rise to $E_{6}$ unifying groups, these $(2,0)$ theories give the more attractive $S O(10)$ and $S U(5)$ gauge groups, with the standard grand unification representations [14]. In addition, these theories have the advantage over the other methods of getting $(2,0)$ theories (free fermions, orbifolds with Wilson lines, etc.) of necessarily preserving a complete separation of the hidden and observable sectors-a characteristic which is quite helpful in building viable models. Furthermore, the number of generations in such vacua bears no relation to the Euler number of the base manifold, and hence there are potentially many phenomenologically acceptable constructions. This is to be contrasted with $(2,0)$ vacua built with the method of $[14,15]$ in which the number of generations equals that of the underlying $(2,2)$ theory; using complete intersection Calabi-Yau manifolds, therefore, there is only one interesting example [18]. Third, although the theories we construct are relatively complicated from the geometrical viewpoint, they have a number of features, similar to those found in $(2,2)$ vacua, which indicate that they too will ultimately be found to have a simple conformal field theoretic description. In particular, we show how the massless spectra and their transformation properties under discrete symmetries can be easily determined by monomial manipulations reminiscent of the local ring of functions which arises in $(2,2)$ Landau-Ginzburg theories. Furthermore, we prove a nonrenormalization theorem for the $(2,0)$ models we construct which ensures that the full tree level superpotential is not corrected by world sheet instantons - thus going beyond (for these cases) the known $(2,2)$ nonrenormalization theorem [19]. Beyond the obvious utility of such a result regarding the extraction of physical properties, this nonrenormalization theorem further illustrates how complicated geometry can give rise to simple and well understood properties for the corresponding conformal theory - a correspondence which, as in the $(2,2)$ case, would be quite worthwhile to understand explicitly.

In Sect. 2 we shall describe Calabi-Yau manifolds in weighted projective space. In Sect. 3 we shall consider the $(2,0)$ constructions and include in our discussion the resolution of an important technical oversight in [17]. In Sect. 4 we shall discuss methods for extracting the phenomenology of these $(2,0)$ theories and describe the nonrenormalization theorems which they respect. In Sect. 5 we shall give our conclusions. 


\section{2. $(2,2)$ Superconformal Models in Weighted Projective Space}

Recently, a largely unexplored class of Calabi-Yau manifolds - certain complete intersection varieties in weighted projective space ${ }^{1}$ have been proposed and argued $[9,10]$ to correspond to the exactly soluble superconformal models comprised of tensor products of members from the $N=2$ discrete series constructed by Gepner. Recall that weighted projective space, $W C P_{k_{1}, \ldots, k_{N+1}}^{N}$, is a generalization of ordinary projective space in which we adopt the equivalence relation on homogeneous coordinates,

$$
\left[z_{1}, \ldots, z_{N+1}\right] \sim\left[\lambda^{k_{1}} z_{1}, \ldots, \lambda^{k_{N+1}} z_{N+1}\right]
$$

for $\lambda$ a complex parameter and positive integers ("weights") $k_{1}, \ldots, k_{n+1}$. A homogeneous polynomial of degree $d$ in $W C P^{N}$ is one which satisfies $P\left(\lambda^{k_{1}} z_{1}, \ldots, \lambda^{k_{N+1}} z_{N+1}\right)=\lambda^{d} P\left(z_{1}, \ldots, z_{N+1}\right)$. (Alternatively, one can think of a weighted projective space as the quotient of $C P^{N}$ by the diagonal group action $Z_{k_{1}} \times \cdots \times Z_{k_{N+1}}$ ). A complete intersection $M$ of dimension $D$ is an algebraic variety (the common zero locus of homogeneous polynomials in $Q_{i}, i=1, \ldots,(N-D)$ in $W C P^{N}$ ) for which

$$
d Q_{1} \wedge d Q_{2} \cdots \wedge d Q_{(N-D)}
$$

does not vanishes on $M$.

Members from the $N=2$ discrete series are labelled by an integer $P_{i}$ and we denote a tensor product of $r$ such theories by $\left(P_{1}, \ldots, P_{r}\right)$. In particular, for $c=9$ theories (corresponding to four extended spacetime dimensions) constructed with five or fewer minimal theories using the affine modular invariant, Gepner's construction corresponds to the Calabi-Yau hypersurface $\sum_{i=1}^{5} z_{i}^{P_{i}+2}=0$ in

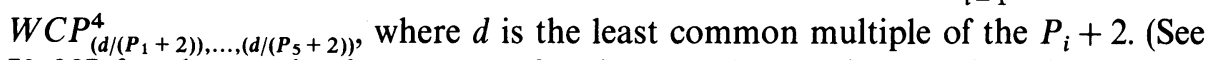
$[9,23]$ for the precise form to use for the $D$ and expectional $E$ invariants.) The proof of equivalence with Gepner's theories relies on a simple path integral argument which we briefly review. For simplicity, consider one of Gepner's theories of the form $\left(P_{1}, \ldots, P_{5}\right)$ using the affine invariant in each theory ${ }^{2}$. The corresponding Landau-Ginzburg theory has superpotential $W=\sum \Phi_{i}^{P_{i}+2}$. By appealing to the universality properties of the renormalization group flow and the nonrenormalization of $W$, we can, at least to leading order, ignore the $D$ terms in the functional integral as they correspond to irrelevant operators. The essential point of [9], then, is the equivalence of the following three statements:

1. The central charge $c=\sum c_{i}$ of the combined minimal models equals 9 .

2. Viewed as the defining equation for an algebraic hypersurface in a uniquely determined weighted projective space, $W=0$ cuts out a manifold (possibly after resolution) of vanishing first Chern class-i.e. a Calabi-Yau manifold.

\footnotetext{
1 For some work that has been done on these manifolds see [20-22]

2 The examples which are not of this sort may be treated similarly - see [9] for details
} 
3. The two-dimensional functional integral with Lagrangian specified by $W$, after a suitable change of variables, gives rise to a multiplicand and $\delta(W)$, which can be interpreted as restricting the (scalar components) of the $\Phi_{i}$ to the above mentioned Calabi-Yau manifold.

Thus, Gepner's construction, which is characterized by the first statement above, manifestly gives rise to string propagation on Calabi-Yau manifolds. Furthermore, the "generalized GSO projection" employed by Gepner to ensure a spacetime supersymmetric spectrum corresponds to orbifolding the initial Landau-Ginzburg theory $[9,10]$. From the point of view in [9], this quotienting arises via the requirement that the change of variables mentioned in the third statement be well defined.

As is familiar from the case of ordinary projective space, the Calabi-Yau condition of vanishing first Chern class is realized by appropriately choosing the degrees of the homogeneous polynomials which define the manifold. The explicit condition on the degrees is most easily derived by considering the adjunction formula for the Chern classes of a variety $K$ in $W C P_{k_{1}, \ldots, k_{N+1}}^{N}$ given by the vanishing locus of homogeneous equations of degrees $d_{1}, \ldots, d_{N-3}$ :

$$
c(K)=\frac{\left(1+k_{1} J\right) \wedge \cdots \wedge\left(1+k_{N+1} J\right)}{\left(1+d_{1} J\right) \wedge \cdots \wedge\left(1+d_{N-3} J\right)}
$$

where $J$ is the Kahler form of $K$. By expanding this formula and extracting the term linear in $J$, we see that the equation for vanishing first Chern class is simply

$$
\sum_{i=1}^{N-3} d_{i}=\sum_{j=1}^{N+1} k_{j}
$$

This generalizes the case of ordinary projective space in which the right-hand side is simply $N+1$. We therefore see the potential for a wealth of new possibilities. It is a simple matter to generalize these considerations to varieties defined in products of weighted projective spaces. Vanishing of the first Chern class then requires (2.4) to hold in each weighted projective space factor, with the $d_{i}$ 's now denoting the degree of homogeneity in the variables of the weighted projective space factor under consideration.

Beyond allowing us to determine the conditions which must be met in order to have a manifold of vanishing first Chern class, (2.4) also provides a means of determining the Euler characteristic of manifolds so constructed. (Recall that the Euler number determines the number of generations in the effective four dimensional theory [1].) For a smooth manifold, the Euler number can be calculated by integrating the third Chern class over the manifold. We can determine the third chern class by expanding (2.4) and picking out the term which is cubic in the Kahler form $J$. Generically, though, varieties constructed in weighted projective space are not smooth but rather are singular. The reason for this is clear: the equivalence relation (2.1) generally has nontrivial fixed points. By fixed points in the context, we are referring to subspaces of the ambient $C^{N+1}$ which are pointwise fixed by nontrivial choices of $\lambda$. If the variety defined in a chosen weighted projective space intersects these singular subspaces, it too will be singular. 
This discussion leads us to two important questions. First, if we choose the degrees of the polynomials to meet (2.4), then are we assured that any singularities inherent to our variety can be resolved without spoiling the condition of vanishing first Chern class? Second, assuming we can resolve the singularities, how does this effect the value of the Euler characteristic? A rigorous treatment of the first question is presently underway [24] and seems to indicate that the answer is yes, provided we choose our defining polynomials $P_{1}, \ldots, P_{N-3}$ so as to meet the transversality constraint (2.2). We can also address this question by a simple physical argument. In [23] is was argued that given a Landau-Ginzburg theory with quasihomogeneous superpotential $W$ (with positive conformal weights) such that $W$ has an isolated critical point at the origin of field space, the renormalization group will cause us to flow to a physically sensible conformal theory. Note that if we identify $W$ with the defining polynomial for a Calabi-Yau hypersurface in $W C P^{4}$, the condition (2.2) is satisfied if $W$ has an isolated critical point at the origin (of $C^{5}$ ). (Choices of $W$ not meeting this condition give rise to Landau-Ginzburg theories with multiple critical points; these are manifested as flat directions [23] which correspond to varieties not satisfying the transversality constraint.) Furthermore, the arguments of [9] show that so long as $d=\sum k_{i}$, we can perform the path integral in the Landau-Ginzburg theory to arrive at the geometric interpretation of a string propagating on an algebraic variety. Thus, since all of the requisite Landau-Ginzburg conditions are met for arriving at a sensible and geometrically interpretable theory, our analysis must be constructing spaces of vanishing first Chern class. Thus, since these singular points are not disconnected in their respective moduli space, we expect that we should be able to resolve, without ruining the Calabi-Yau structure, the singularities in all of the minimal model constructions.

A general answer to the second question listed above will also be given in $[24]^{3}$; for now we are content with the procedure based upon the work of [26] as applied in [9]. Namely,

$$
\chi\left(K_{\text {resolved }}\right)=\int c_{3}(K)-\sum_{i} \chi\left(\mu_{i}\right) / N_{i}+\sum_{i} N_{i} \chi\left(\mu_{i}\right)
$$

where $\mu_{i}$ are the fixed point sets under a common $Z_{N_{i}}$ factor in the weights which is responsible for the singularities. In this expression, we compute the third Chern class, as discussed above, by making use of the adjunction formula. In applying this formula to examples complicated by the existence of overlapping singularities, one must ensure that one does not overcount in the right-hand side. (For explicit examples of the use of this formula, the reader is referred to [9] and to Table 1.)

At first sight it appears that we have at our disposal an enormous number of Calabi-Yau constructions even in a single weighted projective space such as $W C P_{k_{1}, \ldots, k_{5}}^{4}$. So long as we meet the transversality constraint (2.2) and choose our defining equation such that its degree $d$ is equal to the sum of $k_{i}$, then we have a

${ }^{3}$ Recently, Vafa [25] has given a formula for all such theories realizable by Landau-Ginzburg effective actions 
Calabi-Yau manifold. To appreciate this remark, one should contrast it with the situation which arises in ordinary projective four space, $C P^{4}$. The condition of vanishing first Chern class requires that the degree of the defining equation is five, i.e., there is only one allowed choice. This newfound freedom in the weighted projective space case, though, is tempered by the need to meet the transversality constraint - a constraint which is mild in the case of ordinary projective spaces but which is quite restrictive in the case of weighted projective spaces. The essential difference between the ordinary and the weighted projective space constructions in this regard is that for a given degree equation there are far fewer polynomial realizations in weighted projective space. For example, in the simplest case of Fermat hypersurfaces $\sum_{i=1}^{5} z_{i}^{n_{i}}$ of degree $d$, the $n_{i}$ are equal to $d / k_{i}$, and hence the latter must be integral. For ordinary projective spaces with $k_{i}=1$, this constraint is trivial. Similar considerations restrict the possible form of the most general homogeneous polynomials of a given degree in a chosen weighted projective space. This additional restrictiveness in the weighted case makes it more difficult to meet (2.2).

In constructing new examples we restrict attention, for the moment, to Calabi-Yau hypersurfaces in $W C P^{4}$. As discussed, the requirements of transversality and $d=\sum k_{i}$ must both be met in order to have a valid construction. It seems easiest to ensure transversality first (as we will do in a moment) and then determine the sets of weights which can realize $d=\sum k_{i}$. To do this, we make use of the work done in [27] on the partial classification of catastrophic functions with isolated singularities. Such quasihomogeneous functions are characterized by the fact that their derivatives only vanish at the origin. This is precisely what we want, as the origin is excluded from projective space, and hence such functions necessarily meet the transversality constraint. Since we are working in $W C P^{4}$, we are interested in such functions which involve five variables. Unfortunately, such a complete classification is not worked out in [27]; rather, only the case of functions of three variables is treated exhaustively. Taking over these partial results, we are led to consider a simple class of examples which meet (2.2): namely, hypersurfaces whose defining equation $P$ is composed of sums of terms each of which is of the form

$$
z_{i}^{d_{i} ;} \quad z_{i}^{d_{i}}+z_{i} z_{j}^{d_{j} ;} \quad z_{i}^{d_{i}} z_{j}+z_{j}^{d_{j}} z_{k}+z_{k}^{d_{k}} z_{i}
$$

with no overlapping indices between these terms as summands in $P$ (see Table 1 for examples). As mentioned, from the point of view of catastrophe theory [27] the terms of the form (2.6) are singular functions of one, two and three variables with isolated singularities at the origin (in affine space). Most of Gepner's constructions correspond $[9,10]$ to $P$ composed of the subset of terms in (2.6) which are modality zero singular functions, which yield to an $A-D-E$ classification [27]. Landau-Ginzburg potentials which are not composed of modality zero singular functions give rise to superconformal theories which are not in the $N=2$ minimal series (nonminimal theories). This simple generalization beyond the minimal model class (2.6), gives rise to thousands of examples. For illustration we list of few of these examples in the first part of Table 1. One can 
also go beyond hypersurfaces. For instance, we can construct codimension two varieties in $W C P^{5}$. In Table 1 we also list a few (nonsingular) examples of Calabi-Yau manifolds of this sort ${ }^{4}$. More generally, we can consider complete intersections in products of weighted spaces. For illustration, the final example in Table 1 is of this form.

From the Landau-Ginzburg point of view, the varieties in Table 1 which are defined by single equation are realized by choosing a superpotential of the same form as the equation and following the path integral prescription of [9] as reviewed earlier. The codimension two varieties can also be realized as Landau-Ginzburg theories by introducing a Lagrange multiplier as we now describe. For concreteness, consider the first example in Table 1 which is embedded in $W C P^{5}$. From a Landau-Ginzburg potential,

$$
\Phi_{1}^{2}+\Phi_{2}^{2}+\sum_{n=3}^{6} \Phi_{n}^{4}+\Lambda\left(\Phi_{1}^{2}+2 \Phi_{2}^{2}+\sum_{n=3}^{6} n \Phi_{n}^{4}\right)
$$

which we denote by $P_{1}+\Lambda P_{2}$. In essence, we view $\Lambda$ as a superfield with trivial scaling dimension. With this potential we evaluate the path integral

$$
\int\left[d \Phi_{1}\right] \cdots\left[d \Phi_{6}\right][d \Lambda] \exp \left\{\int d^{2} z d^{2} \theta\left(P_{1}+\Lambda P_{2}\right)\right\} .
$$

We do this by changing variables to $\xi_{1}=\Phi_{1}^{2}, \xi_{2}=\Phi_{2} / \Phi_{1}, \xi_{n}^{4}=\Phi_{n}^{4} / \Phi_{1}^{2}$ for $n=3,4,5,6$ and $\xi_{7}=\Lambda \Phi_{1}^{2}$. It is straightforward to check that this change of variables has constant Jacobian (precisely due to the inclusion of $\Lambda$ ) and that the $\xi_{1}$ and $\xi_{7}$ integrations immediately give a factor of

$$
\delta\left(P_{1}\right) \delta\left(P_{2}\right)
$$

(in inhomogeneous $W C P^{5}$ coordinates), thus constraining (an orbifold of) the Landau Ginzburg theory to live on the algebraic variety. A similar procedure works for the other examples. In particular, for the $\chi=-156$ example, the Lagrange multiplier $\Lambda$ must have conformal weight $1 / 3$. Only with this inclusion is the central charge of the associated Landau-Ginzburg theory equal to 9; correspondingly, only with such a $\Lambda$ can we apply our path integral argument to arrive at a geometric interpretation of this theory.

Clearly, there are a great many Calabi-Yau manifolds which can be constructed along the lines of Table 1. (Of course, there may be nontrivial identifications which can be made amongst such manifolds). Since ordinary projective spaces constitute a special case of these more general weighted spaces, we see that weighted projective spaces provide a unifying framework within which a great many classical vacua, both singular and smooth, reside. In fact, it may be that all simply connected Calabi-Yau manifolds are biregular to complete intersection constructions in products of weighted projective spaces [28]. Even so, the procedure for constructing these manifolds is defined in a narrow enough way to admit a systematic analysis. One interesting question, as briefly mentioned above, is to understand in more

\footnotetext{
${ }^{4}$ After constructing these examples we became aware of [22] in which these three examples (which are the only nonsingular examples of the Fermat type in $W C P^{5}$ ) were first constructed
} 
Table 1. A few examples of Calabi-Yau compactifications in weighted projective space which are realizable as (non-minimal model) Landau-Ginzburg theories

\begin{tabular}{lll}
\hline Defining Equations & Ambient Space & $\chi$ \\
\hline$z_{1}^{14}+z_{2}^{14}+z_{3}^{7}+z_{4}^{4} z_{3}+z_{5}^{2} z_{4}=0$ & $W C P_{1,1,2,3,7}^{4}$ & -260 \\
$z_{1}^{10}+z_{2}^{5}+z_{3}^{5}+z_{4}^{5}+z_{5}^{3} z_{1}=0$ & $W C P_{1,2,2,2,3}^{4}$ & -144 \\
$z_{1}^{22}+z_{2}^{11}+z_{3}^{7} z_{1}+z_{4}^{4} z_{2}+z_{5}^{2}=0$ & $W C P_{1,2,3,5,7,11}^{4}$ & -196 \\
$z_{1}^{20}+z_{2}^{20}+z_{3}^{5} z_{4}+z_{4}^{4}+z_{5}^{2}=0$ & $W C P_{1,1,3,5,10}^{4}$ & -292 \\
$z_{1}^{22}+z_{2}^{22}+z_{3}^{11}+z_{4}^{3} z_{1}+z_{5}^{2}=0$ & $W C P_{1,1,2,7,11}^{4}$ & -420 \\
$z_{1}^{2}+z_{2}^{2}+\sum_{n=3}^{6} z_{n}^{4}=0$ & $W C P_{2,2,1,1,1,1}^{5}$ & -144 \\
$z_{1}^{2}+2 z_{2}^{2}+\sum_{n=3}^{6} n z_{n}^{4}=0$ & & \\
$z_{2}^{2}+z_{3}^{2} \sum_{n=3}^{6} z_{n}^{4}=0$ & & \\
$z_{1}^{2}+2 z_{2}^{3}+3 z_{3}^{3}+\sum_{n=4}^{6} n z_{n}^{6}=0$ & $W C P_{3,2,2,1,1,1}^{5}$ & -156 \\
$z_{1}^{2}+z_{2}^{2}+z_{3}^{3}+z_{4}^{3}+z_{5}^{6}+z_{6}^{6}=0$ & & \\
$z_{1}^{2}+2 z_{2}^{2}+3 z_{3}^{3}+4 z_{4}^{3}+5 z_{5}^{6}+6 z_{6}^{6}=0$ & & \\
$z_{1}^{2}+\sum_{i=2}^{4} z_{1}^{4}=0$ & & \\
$x_{1}^{3} z_{2}+x_{2}^{3} z_{3}+x_{3}^{3} z_{4}=0$ & & \\
\hline
\end{tabular}

precise mathematical terms the conditions under which the quotient singularities which we encounter are harmless [24]. The argument based upon the LandauGinzburg interpretation given above implies that we should be able to resolve any singular variety meeting our hypotheses to a smooth Calabi-Yau manifold. It would also be interesting to seek out the new $N=2$ (nonminimal) superconformal models to which these Landau-Ginzburg theories correspond. Presumably, some of these correspond to the coset models of Kazama and Suzuki [29]. Another question involves extending the work in $[30,31]$ to search these manifolds for physically interesting examples. For example, does the surprising paucity of three generation examples persist in this broadened class of manifolds? In fact, since we are broadening our perspective to include manifolds with quotient singularities, it would be of use to extend the work of $[30,31]$ into the realm of (sufficiently well behaved) fixed point group actions both in the context of ordinary and weighted projective space varieties. At least for those weighted projective space Calabi-Yau manifolds corresponding to Gepner's construction, this should be straightforward to do from the conformal field theory point of view. That is, we can mod through the initial Gepner theory by any symmetry (more precisely, any non-R-symmetry) and ascertain the resulting spectrum as discussed in [7]. Finally, 
an interesting and important question is whether the connectivity of the moduli space for ordinary complete intersection Calabi-Yau manifolds found and discussed in [32] can be extended to these complete intersections in weighted spaces. We shall not discuss these issues here, but rather concentrate on one interesting application of the varieties constructed in this manner-the construction of $(2,0)$ string compactifications.

\section{3. $(2,0)$ Compactifications in Weighted Projective Space}

The compactifications discussed in the last section have $(2,2)$ world sheet supersymmetry as we were implicitly embedding the spin connection in the gauge group [1] to ensure anomaly freedom. (From the two-dimensional point of view, we were making use of an $E_{8} \times S O(10) \mathrm{Kac}-$ Moody algebra in the left moving sector of the heterotic string). However, the preservation of $N=1$ spacetime supersymmetry, which is crucial to our being certain that our putative ground state is a solution to the string equations of motion beyond lowest order sigma model and string loop expansion considerations, only requires that the world sheet theory have $(2,0)$ supersymmetry [33-35]. The conditions for $(2,0)$ supersymmetry were studied in $[33,14]$ and the requirements are as follows: the compact manifold $K$ must be a Calabi-Yau manifold and the vacuum gauge bundles $V_{1}$ and $V_{2}$ (to which the left moving fermions $\lambda^{a}$ couple) must be the direct sums of stable holomorphic vector bundles satisfying

$$
\begin{gathered}
c_{1}\left(V_{1}\right)=c_{1}\left(V_{2}\right)=0, \\
c_{2}(T)=c_{2}\left(V_{1} \oplus V_{2}\right) .
\end{gathered}
$$

In this expression, $c_{1}$ and $c_{2}$ are the first and second Chern classes, respectively. The well studied scenario of $(2,2)$ compactifications corresponds to choosing $V_{1}$ to be the tangent bundle and $V_{2}$ to be trivial.

The reasons for studying these more general solutions to (3.1),(3.2) are clear. First, from the point of view of investigating the structure of the space of classical string vacua (e.g. the classification program), there is no reason at all to restrict attention to the $(2,2)$ subclass. Second, from the point of view of phenomenology, these theories have exceptional promise. As originally emphasized in [14], a number of the generic phenomenological problems of $E_{6}$ based $(2,2)$ models can be solved by $(2,0)$ theories as the latter offer the possibility of lower rank unifying groups. The essential idea here [14], is that our bundle $V_{1}$ can have, for example, structure group $S U(4)$ or $S U(5)$ (as opposed to the $S U(3)$ structure group of the tangent bundle) and hence give rise to $S O(10)$ or $S U(5)$ four dimensional unifying groups. Whereas the matter multiplets in $(2,2)$ models are in the 27 of $E_{6}$, these $(2,0)$ theories give rise to the 16 's of $S O(10)$ or the $(5+\overline{10})$ 's of $S U(5)$ familiar from conventional grand unification. After flux breaking (if the base manifold is multiply connected) these gauge groups can be broken to the standard model (with a possible extra $U(1)$ in the $S O(10)$ case) [36]. In addition to yielding models with more economical gauge groups, there is another important property of $(2,0)$ theories: the number of generations is given by half of the third Chern number of the 
vacuum gauge bundle in the observable sector-a number which need not have any relation to the Euler characteristic of the base manifold. Thus, it may be possible to go beyond the "uniqueness" results of [30,31], which essentially proved that there is only one three generation $(2,2)$ example in the class of complete intersection Calabi-Yau manifolds in products of ordinary projective space, and construct new three generation examples.

In [16], however, it was argued that although these attractive $(2,0)$ theories are solutions to the classical string equations of motion to all orders in sigma model perturbation theory [14], they are destabilized by world sheet instantons. The essential difference between a $(2,2)$ and a $(2,0)$ theory in this regard is that the additional world sheet supersymmetry enjoyed by the former ensures the presence of left moving fermion zero modes in an instanton background. These fermion zero modes prevent the generation of a purely dilatonic contribution to the superpotential which would destabilize the theory. The authors of [16] argued that the generic $(2,0)$ theory will not have such zero modes and hence will be destabilized.

In practice, however, it appears that it is not too difficult to avoid this potential destabilization. In fact, two general mechanisms [17, 15] (see also [37]) have been proposed to accomplish this. One mechanism is based on the proposal in [14] to construct $(2,0)$ theories from $(2,2)$ theories by holomorphically deforming $T \oplus O$ into a stable rank four bundle. The ability to carry out such a deformation relies on the existence of certain exactly flat directions in the superpotential. As shown in [15], these flat directions can be guaranteed (in some compactifications) by spacetime $R$-symmetries. Thus, the lifting of flat directions in the superpotential by world sheet instantons indicated in [16] is prevented by a discrete symmetry. (Note that the number of generations in $(2,0)$ theories constructed in this way is equal to that of the initial $(2,2)$ theory.)

Another mechanism for avoiding the destabilization by world sheet instantons was presented in [17]. The essential idea in this approach is that the existence of fermion zero modes, which is ensured in $(2,2)$ theories by the left moving world sheet supersymmetry, can also be guaranteed by geometrical considerations. In particular, it was shown in [17] that if the vacuum gauge bundle is nontrivial when restricted to any holomorphic curve of genus zero on $K$, then the $(2,0)$ theory is not destabilized by world sheet instantons. Explicitly, this condition of nontriviality implies, by the Grothendieck theorem, that when we express the restriction of the vacuum gauge bundle $V$ to any $C P^{1}$ as a direct sum of line bundles $O(a)$

$$
\left.V\right|_{C P^{1}}=O\left(a_{1}\right) \oplus \cdots \oplus O\left(a_{r}\right)
$$

that not all of the $a_{i}$ vanish. (Recall that (3.1) implies that the sum of the $a_{i}$ does vanish). In other words, if we augment the conditions (3.1),(3.2) (which are the conditions to have a solution in sigma model perturbation theory) by the one nonperturbative condition of $(3.3)$ being nontrivial, then the $(2,0)$ theory is stable with respect to world sheet instantons. As mentioned in the introduction, an interesting question from the point of view of both classifying string vacua and also understanding string phenomenology is whether there is an abundance or a paucity of examples meeting these conditions. We now turn to this question. 
The first step in finding explicit examples which meet these criteria is the construction of stable holomorphic vector bundles. Stability of a bundle $V$ is the requirement that the normalized first Chern number of $V$ is greater than that of any sub-bundle (more precisely, subsheaf). Intuitively, this is the requirement that the curvature of $V$ is greater than that of any of its sub-bundles. This requirement arises as one of the conditions for having a solution to the classical string equations of motion through the need to solve the equation $[1,14] g^{i \bar{j}} F_{i \bar{j}}=0$, where $g_{i \bar{j}}$ is the Calabi-Yau metric and $F_{i \bar{j}}$ is the curvature of the vacuum gauge bundle. It was shown in [38] that a necessary and sufficient condition to solve this equation is that $V$ be the direct sum of stable bundles. Stability is not a very difficult requirement to meet, but given a bundle, it is somewhat difficult to verify. There is, however, a simple algorithm for generating stable bundles due to [39]. We will have recourse momentarily to use this algorithm, so we now briefly describe it. For more details see on this application of [39] see [17].

To construct a stable holomorphic bundle $E$ of rank $r$ over a Kahler manifold $K$ one must choose a smooth effective divisor $D$ of $K$ and form the following two exact sequences:

$$
\begin{gathered}
0 \rightarrow O_{D}(-n J) \rightarrow O_{D}^{\oplus r} \rightarrow F \rightarrow 0, \\
0 \rightarrow E \otimes O_{M}(J) \otimes L_{D}^{-1} \rightarrow O_{M}^{\oplus r} \rightarrow F \rightarrow 0,
\end{gathered}
$$

where $J$ is the integral cohomology class of the Kahler form on $K$ (we assume that $K$ is projective algebraic), $L_{D}$ is the line bundle determined by the divisor $D$ (with first Chern class $[D]$ ), $n \geqq r$ is an integer, $O_{A}(a J)$ denotes the $a^{\text {th }}$ power of the hyperplane bundle (the line bundle associated to the Kahler class) over $A$ (for negative $a$ this refers to the dual bundle), and the first map in (3.4) is given by choosing $r$ linearly independent sections $s_{i} \in H^{0}\left(D, O_{D}(n J)\right)$ not simultaneously vanishing at any point of $D$. In what follows, we shall sometimes write $O_{A}(a)$ for $O_{A}(a J)$. Sequence (3.4) defines $F$ and (3.5) defines the stable bundle $E$. As shown in [17], the Chern classes of $E$ are as follows:

$$
\begin{aligned}
& c_{1}(E)=[D]-r J, \\
& c_{2}(E)=\frac{r(r-1)}{2} J^{2}+(n-r+1) J[D], \\
& c_{3}(E)=\frac{r(r-1)(r-2)}{6} J^{3}+\frac{2(n+1)^{2}-r(2 n-r+3)}{2} J^{2}[D] .
\end{aligned}
$$

Given a Calabi-Yau manifold $K$, this stable bundle algorithm can be used to try to meet the constraints in (3.1) and (3.2). An example of this sort was presented in [17] for the Calabi-Yau manifold $Y_{4 ; 5}$. This manifold has $c_{2}=10 \mathrm{~J}^{2}$, and hence if we take $n=r=4$ we meet the required conditions on the Chern classes of $E$.

There is, of course, the additional constraint (3.3) which must also be met. In [17] it was shown that if divisor $D$ is chosen so as to intersect the $C P^{1}$ 's on $K$ in points, then the bundle $E$ necessarily satisfies (3.3) (with some $a_{i}$ nonzero). For the case of $Y_{4 ; 5}$, this was explicitly demonstrated for the 375 lines identified in [16]. It turns out, however, that these 375 lines do not exhaust the complete set as there 
are 2875 lines on the generic quintic hypersurface in $C P^{4}$ [40]. (We note that this number includes the possibility of some lines having multiplicity greater than one, however, this will not be important to us.) There are a couple of ways we can deal with this technical deficiency of [17] due to these additional lines; we choose to show that our divisor $D$ can be chosen to meet them in points as well. ${ }^{5}$ In fact, the argument is quite simple, so we phrase it for the general case.

Let $F_{1}(K)$ be the Fano variety of lines [42] for a Calabi-Yau threefold $\mathrm{K}$. Consider the incidence correspondence $\Sigma=\{\delta \times l: l \in \delta\} \in \Delta \times F_{1}(K)$, where $\Delta$ is the set of divisors of $K$ in a chosen divisor class. There are two natural projections: $\pi_{1}: \Sigma \rightarrow \Delta$ and $\pi_{2}: \Sigma \rightarrow F_{1}(K)$. The fiber $f_{1}$ of the first projection consists of the set of lines contained on a given divisor $\delta$ and the fiber $f_{2}$ of the second projection consists of the set of divisors containing a given line $l$. To prove that we can chose a divisor $D \in \Delta$ intersecting all of the lines in $F_{1}(K)$ in points, we simply need to prove that the first projection is not surjective. This is readily done by counting dimensions: if $\operatorname{dim}\left(F_{1}(K)\right)+\operatorname{dim}\left(f_{2}\right)$ is less than $\operatorname{dim} \Delta$, then we are done. On the generic ${ }^{6}$ complete intersection Calabi-Yau threefold, $\operatorname{dim}\left(F_{1}(K)\right)$ is zero. (The reader can easily convince himself of this fact by a simple dimension count, analogous to the one used in this argument.) Thus if we choose a generic complete intersection Calabi-Yau three fold $K$, we can always choose a divisor $D$ intersecting the lines on $K$ in distinct points. More generally, for an arbitrary Calabi-Yau threefold, $\operatorname{dim} F_{1}(K)$ may be positive. This dimension counting argument, though, goes through unhindered if the degree of the divisor $D$ is sufficiently large (so that the dimension of $\Delta$ is sufficiently large-typically degree two will suffice). We see quite generally, therefore, that $D$ can be chosen to intersect the lines on $K$ in distinct points. The stable bundle construction requires that $D$ be smooth as well. Since we now see that the condition of the divisor intersecting lines in points is an open condition (and, of course, smoothness is an open condition) and two nonempty open sets (in the Zariski topology) always intersect, we can choose $D$ to be smooth as well. Finally, this argument can readily be extended to higher degree holomorphic curves. ${ }^{7}$ Thus, $(2,0)$ models constructed as above yield stable string vacua.

In [17], only one explicit example of $(2,0)$ theory meeting all of the necessary constraints was constructed. The example is built from the rank four bundle $E$ and $Y_{4 ; 5}$ (described after (3.8)) and has an $S O(10)$ structure group with 100 generations of 16's. The number of generations is computed from the third Chern number of the bundle $E$, which by (3.8) is $40 \mathrm{~J}^{3}$. One should note that all of the Chern classes of this bundle are the same as that for the cotangent bundle of $Y_{4: 5}$, and hence one might suspect that all we have constructed is a deformation of the underlying $(2,2)$ theory. More precisely, the data computed from Eqs. (3.6), (3.7),

\footnotetext{
5 Alternatively, it can be shown that a stable bundle constructed by the Maruyama method is nontrivial over any holomorphic curve of genus zero even if the divisor $D$ does not meet the curve in distinct points [41]

${ }^{6}$ By generic in this context, one refers to an element of an open set of a variety which serves as a parameter space for the objects under consideration

${ }_{7}$ We thank J. Harris for this point, and for discussions on the arguments of this paragraph
} 
(3.8) are consistent with $E$ being a holomorphic deformation of $T^{*} \oplus O$. In fact, Dine and Seiberg have shown, using the discrete symmetries of $Y_{4 ; 5}$, that there are exact flat directions in the superpotential which permit such a deformation [15].

We will not try to prove, one way or the other, whether the example presented in [17] is a deformation of the standard $(2,2)$ theory. Rather, we present new examples of $(2,0)$ models constructed by the method described above which are definitively not deformations of $(2,2)$ theories. If all such $(2,0)$ theories were deformations of $(2,2)$ theories, one might be able to classify all possibilities by concentrating just on the latter. Furthermore, deformations of $(2,2)$ theories, as mentioned, preserve the number of generations. Hence, if one wants to construct a three generation $(2,0)$ model, for instance, from an underlying $(2,2)$ theory, there is essentially a unique $[30,31]$ choice for the initial $(2,2)$ theory. Some work along these lines can be found in [18]. The construction of $(2,0)$ models by the means described in [17] and above, though, has the potential to yield models in which the number of generations is independent of the corresponding number found in the associated $(2,2)$ theory.

At first sight, however, it is not that easy to construct new examples. The problem is that the value of the second Chern class for a number of the simplest Calabi-Yau manifolds (say, those embedded as complete intersections in a single nonweighted projective space - the so-called $Y$ series) is too small for Eq. (3.2) to be met by a rank four (or higher) bundle with $c_{2}$ given by (3.7). The varieties in weighted projective space discussed in the last section provide us with a way around this difficulty ${ }^{8}$. We explore this possibility below and show that we can construct a number of new examples - many of which are not deformations of the underlying $(2,2)$ theory.

To avoid unnecessary complexity, we restrict our attention to Calabi-Yau manifolds in a single weighted projective space which avoid all of the ambient space singularities. The manifolds of interest were originally constructed in [21] and [22]. In particular, consider [21] three Fermat hypersurfaces of degrees 6, 8 and 10 in $W C P_{1,1,1,1,2}^{4}, W C P_{1,1,1,1,4}^{4}$ and $W C P_{1,1,1,2,5}^{4}$ which we call $Y_{W 4 ; 6}, Y_{W 4 ; 8}$ and $Y_{W 4 ; 10}$ respectively. We will also make use of the last three examples in Table 1, $Y_{W 5 ; 4,4}, Y_{W 5 ; 6,4}$ and $Y_{W 5 ; 6,6}$. The Euler numbers and second Chern classes of these manifolds are computed as in the last section. The larger values of $c_{2}$ gives us more possibilities for meeting (3.2); in Table 2 we list the various values of $n$ and $r$ (as an ordered pair in that order) which allow us to meet this constraint. In this table, $V_{1}$ and $V_{2}$ are the observable and hidden sector gauge bundles and $G$ denotes the observable four dimensional gauge group.

For all cases, we choose $[D]=r J$ as this is the only way to meet the integrability condition [38] $\int c_{1}(E) \wedge J^{2}=0$ on a manifold with $h^{1,1}=\operatorname{dim} H^{1}\left(T^{*}\right)=1$. We see that only for the first three examples does the value of $c_{2}$ force us to a unique choice for the vacuum gauge bundle. The other three examples give rise to a

\footnotetext{
${ }^{8}$ We also note that for the purposes of constructing examples, it is also worthwhile to consider bundles $E$ with rank three - that is, $(2,0) E_{6}$ theories. It is not hard to show [43] that the $Y$-series give rise to one such example-a $(2,0)$ theory on $Y_{5 ; 3,3}$, the vanishing locus of two cubics in $C P^{5}$. To construct this model, we take $n=r=3$; we note that it gives rise to 90 generations
} 
Table 2. Examples of $(2,0)$ Models in Weighted Projective Space

\begin{tabular}{llrlllr}
\hline Manifold & $c_{2}$ & $\frac{1}{2}|\chi|$ & $V_{1}$ & $V_{2}$ & $\mathrm{G}$ & Generations \\
\hline$Y_{W 5 ; 4,4}$ & $10 J^{2}$ & 72 & $(4,4)$ & $O$ & $S O(10)$ & 80 \\
$Y_{W 4 ; 6}$ & $14 J^{2}$ & 102 & $(5,4)$ & $O$ & $S O(10)$ & 102 \\
$Y_{W 5 ; 6,4}$ & $16 J^{2}$ & 78 & $(4,4)$ & $(3,3)$ & $S O(10)$ & 40 \\
$Y_{W 4 ; 8}$ & $22 J^{2}$ & 148 & $(7,4)$ & $O$ & $S O(10)$ & 148 \\
$Y_{W 5 ; 6,6}$ & & & $(4,4)$ & $(5,3)$ & $S O(10)$ & 40 \\
$Y_{W 4 ; 10}$ & $22 J^{2}$ & 60 & $(7,4)$ & $O$ & $S O(10)$ & 74 \\
& & & $(4,4)$ & $(5,3)$ & $S O(10)$ & 20 \\
& $34 J^{2}$ & 144 & $(4,4)$ & $(3,3) \oplus(6,4)$ & $S O(10)$ & 20 \\
& & & $(4,4)$ & $(5,3) \oplus(5,3)$ & $S O(10)$ & 20 \\
& & & $(4,4)$ & $(7,3) \oplus(3,3)$ & $S O(10)$ & 20 \\
& & & $(4,4)$ & $(9,3)$ & $S O(10)$ & 20 \\
& & & $(5,4)$ & $(4,4) \oplus(4,4)$ & $S O(10)$ & 34 \\
& & & $(5,4)$ & $(5,4) \oplus(3,3)$ & $S O(10)$ & 34 \\
& & & $(10,4)$ & $(6,5)$ & $S O(10)$ & 34 \\
& & & $(5,5)$ & $(5,4) \oplus(4,3)$ & $S O(10)$ & 185 \\
& & & $(6,5)$ & $(5,4)$ & $S U(5)$ & 35 \\
& & & $(7,5)$ & $(4,3)$ & $S U(5)$ & 80 \\
\hline
\end{tabular}

number of possible solutions. For the first time, a number of these examples necessarily invoke a nontrivial gauge bundle in the hidden sector. For instance, the last example gives rise to an $S U(5)$ gauge theory in the observable sector and an $E_{6}$ theory in the hidden sector. (Of course, the roles of "observable" and "hidden" sectors can be reversed in all of these constructions giving rise to additional models.) In the second and fourth examples, the number of generations in our $(2,0)$ theory is the same as in the associated $(2,2)$ theory, and hence it is at least possible that the former are holomorphic deformations of the latter. The number of generations in the other examples, however, is distinct from that of the $(2,2)$ theory built on the same manifold, so they can not arise via such holomorphic deformations. We thus see that in contrast to previous expectations, we can construct numerous stable $(2,0)$ vacua which are not continuously connected to an underlying $(2,2)$ theory.

It is interesting to note that the number of generations in many of these $(2,0)$ theories is less than in the associated $(2,2)$ theory. Intuitively, the reason for this is that we are meeting the anomaly cancellation requirement (3.1) with the contribution of a nontrivial gauge bundle in the hidden sector. However, only the observable sector part of the total gauge bundle (i.e. $V_{1}$ ) contributes to the observable spacetime spectrum. This subbundle of the full gauge bundle, which necessarily has "smaller" $c_{2}$ than the tangent bundle, often has smaller third Chern number as well.

We also remark that in our analysis of $(2,0)$ theories we have only considered weighted varieties which avoid all of the ambient space singularities. Preliminary work [24] indicates that it should be possible to consider constructions on the numerous weighted varieties which do not have this property. One example of 
this sort [24] allows a vacuum gauge bundle consisting of two identical rank 5 stable bundles. This realizes the amusing possibility [14] of having a "mirror" universe in the hidden sector.

We now briefly turn to a general discussion of extracting phenomenology from these theories

\section{Extracting Phenomenology from $(2,0)$ Theories}

As is familiar from $(2,2)$ theories, in addition to the net number of generations, model building requires knowledge of additional (quasi) topological properties of the compactification. In particular, one needs to understand the cohomology of the vacuum gauge bundle. At the very least, we need the dimensions of $H^{*}(K, V)$-at best, we can find explicit representatives for the nontrivial cohomology (to facilitates the computation of the Yukawa couplings and the determination of discrete symmetry properties). We now show, happily, that the cohomology of the vacuum gauge bundles constructed by the Maruyama prescription is readily computed and admits an explicit representation. This was indicated in [17] for the example constructed on $Y_{4 ; 5}$. We now show that such considerations are general.

Our analysis requires a number of standard facts which we now briefly review. For more details see [42]. Let $L_{K}$ denote the canonical line bundle of a Kahler manifold $K$ (with dual bundle $L_{K}^{-1}$ ) and let $L$ denote any positive line bundle on $K$. The Kodaira Vanishing theorem (KVT) states that $\operatorname{dim} H^{q}\left(K, L \otimes L_{K}^{-1}\right)=0$ for $q \geqq 1$. Kodaira-Serre duality is the general result that $\left.H^{q}(K, E) \cong H^{n-q}\left(K, E^{*} \otimes L_{K}\right)\right)^{*}$, where $E$ is a vector bundle over $K$ and $n$ is the dimension of $K$. Applying Poincaré duality to the Kodaira vanishing theorem implies $\operatorname{dim} H^{q}(K, L)=0$ for $L$ any negative line bundle and $q<n$. Also note that $\operatorname{dim} H^{0}(K, E)=0$ for any stable bundle $E$. Over a manifold of vanishing first Chern class, duality and the stability of $E^{*}$ then gives $\operatorname{dim} H^{n}(K, E)=0$ as well. Finally, if $D$ is a divisor on $K$ and $L_{D}$ its associated line bundle, we have the exact sequence

$$
0 \rightarrow O_{K} \otimes L_{D}^{-1} \rightarrow O_{K} \rightarrow O_{D} \rightarrow 0,
$$

where $O_{K}$ and $O_{D}$ are the structure sheafs of $K$ and $D$, respectively. Having stated the basic results we need, we now turn to the computation of the cohomology of a general Maruyama bundle over a Calabi-Yau three fold $K$.

Let $K$ be a Calabi-Yau three fold and $D$ a smooth positive divisor with $[D]=s J$ with $1<s<n+1$ (with $n$ defined (as before) to be an integer greater than the rank $r$ of the bundle $E$ ). Consider the exact sequence

$$
0 \rightarrow O_{K}(-1) \rightarrow O_{K}(s-1) \rightarrow O_{D}(s-1) \rightarrow 0
$$

and its associated long exact sequence in cohomology. By the KVT for negative line bundles, the only nontrivial cohomology in $H^{*}\left(O_{K}(-1)\right)$ is in the top dimension; we set $\alpha=\operatorname{dim} H^{3}\left(O_{K}(-1)\right)$. By the KVT for positive line bundles (and recalling that the canonical line bundle for a Calabi-Yau manifold is trivial) the only cohomology in $H^{*}\left(O_{K}(s-1)\right)$ is in the lowest dimension, so define $\beta=$ $\operatorname{dim} H^{0}\left(O_{K}(s-1)\right)$. From the long exact cohomology sequence associated to (4.2), we then find that $\operatorname{dim} H^{0}\left(O_{D}((s-1))\right)=\beta, \operatorname{dim} H^{1}\left(O_{D}(s-1)\right)=0$ and 
$\operatorname{dim} H^{2}\left(O_{D}(s-1)\right)=\alpha$. Now consider the exact sequence

$$
0 \rightarrow O_{K}(-n-1) \rightarrow O_{K}(s-n-1) \rightarrow O_{D}(s-n-1) \rightarrow 0 .
$$

Using the KVT as before, the only nontrivial cohomology for the first two terms in this sequence lies in the top dimension and we define $\gamma=\operatorname{dim} H^{3}\left(O_{K}(-n-1)\right)$ and $\delta=\operatorname{dim} H^{3}\left(O_{K}(s-n-1)\right)$. By the long exact cohomology sequence, we then have $\operatorname{dim} H^{0}\left(O_{D}(s-n-1)\right)=0, \operatorname{dim} H^{1}\left(O_{D}(s-n-1)\right)=0$ and $\operatorname{dim} H^{2}\left(O_{D}(s-n-1)\right)=$ $\delta-\gamma$. Now consider the defining sequence (3.4) for the sheaf $F$,

$$
\left.0 \rightarrow O_{D}(s-n-1)\right) \rightarrow O_{D}^{\oplus r}(s-1) \rightarrow F(s-1) \rightarrow 0 .
$$

From the cohomology dimensions above, it is straightforward to compute that $\operatorname{dim} H^{0}(F(s-1))=r \beta, \operatorname{dim} H^{1}(F(s-1))=r \alpha-\delta+\gamma+a, \operatorname{dim} H^{2}(F(s-1))=a$, where $a$ is an integer to be determined momentarily. Finally, we can use these results in the cohomology sequence associated to the defining sequence for the bundle $E$,

$$
0 \rightarrow E \rightarrow O_{K}^{\oplus r}(s-1) \rightarrow F(s-1) \rightarrow 0
$$

in conjunction with the fact, discussed above, that $\operatorname{dim} H^{0}(E)=\operatorname{dim} H^{3}(E)=0$ to find that the only nonzero cohomology for the bundle $E$ is in dimension two ${ }^{9}$, and $\operatorname{dim} H^{2}(E)=r \alpha-\delta+\gamma$. In particular,

$$
H^{2}(E) \cong H^{1}(F(s-1))=\operatorname{ker}\left(H^{2}\left(O_{D}(s-n-1)\right) \rightarrow H^{2}\left(O_{D}^{\oplus r}(s-1)\right)\right) .
$$

Since this map is surjective we have

$$
\operatorname{dim} H^{2}(E)=\operatorname{dim} H^{2}\left(O_{D}(s-n-1)\right)-\operatorname{dim} H^{2}\left(O_{D}^{\oplus r}(s-1)\right) .
$$

The right-hand side of this expression is easily computed by means of the exact sequence (4.1) and Bott's formula [44]. It proves worthwhile, though, to analyse this a bit further. By duality, (4.7) implies that

$$
\operatorname{dim} H^{2}(E)=\operatorname{dim} H^{0}\left(O_{D}(n+1)\right)--\operatorname{dim} H^{0}\left(\left(O_{D}^{\oplus r}(1)\right) .\right.
$$

The nice thing about this expression is that we can compute $\operatorname{dim} H^{2}(E)$ by simple combinatorial reasoning, similar to that which is often applicable in $(2,2)$ theories $[45,46]$. For simplicity, assume that $K$ is a complete intersection in one projective space $C P^{N} . H^{0}\left(O_{D}(p)\right)$ consists of $p^{\text {th }}$ order monomials in the coordinates of $C P^{N}$ less the number, $t$, of those which are rendered trivial by the equations defining $K$ and $D$. We thus have

$$
\operatorname{dim} H^{2}(E)=\frac{(N+n+1) !}{N !(n+1) !}-r(N+1)-t .
$$

Let's apply this formula to the two examples of Maruyama bundles on $Y_{4 ; 5}$ and $Y_{5 ; 3,3}$ described earlier. The former has $N=4, n=4, r=4$ which yields $\operatorname{dim} H^{2}(E)=100$; the latter has $N=5, n=3, r=3$ which gives $\operatorname{dim} H^{2}(E)=90$. Notice that both of these numbers agree with the computation above of $c_{3}(E)$, thus providing a nice check on our results.

\footnotetext{
9 In other words, these constructions give rise to no antigenerations for 27's of $E_{6}, 16$ 's of $S O(10)$ or 10 's of $S U(5)$
} 
It does not take much effort to extend this analysis to the $(2,0)$ theories which we have constructed in weighted projective space. The essential difference in computing the cohomology of these examples lies in the final stage of the calculation when we relate the bundles in (4.8) via (4.1) to line bundles over the ambient space whose cohomology we know by Bott's theorem. The ambient space is now a weighted projective space, so we need to generalize Bott's theorem. This is not difficult and has been done in [47]. For line bundles in weighted projective space, as for those on ordinary projective space, the only nonzero cohomology is in the lowest or highest dimension. For the former we can compute $\operatorname{dim} H^{0}(O(a J))$ (assume a is positive) over $W C P_{k_{1}, \ldots, k_{N+1}}^{N}$ by counting the number of independent monomials of degree $a$ in the $N+1$ coordinates on $W C P^{N}$, where the degree of $z_{i}^{d_{i}}$ is, of course, $k_{i} d_{i}$. This is readily determined by simple combinatorial analysis or by picking out the coefficient of $t^{a}$ in the Poincare polynomial [47] $\prod\left(1-t^{k_{i}}\right)^{-1}$. For $H^{N}(O(b J))$ with $b$ negative, the easiest way to proceed is ${ }^{i}$ to use duality to write $\operatorname{dim} H^{N}(O(b J))=\operatorname{dim} H^{0}\left(O(-b J) \otimes K_{W C P^{N}}\right)$, where $K_{W C P^{N}}$ is the canonical bundle on $W C P^{N}$ and, as discussed, is given by $O\left(-\left(k_{1}+\cdots k_{N+1}\right) J\right)$. Thus, $\operatorname{dim} H^{N}(O(b J))=\operatorname{dim} H^{0}\left(-b-\left(k_{1}+\cdots+k_{N+1}\right) J\right)$, and hence can be computed by the monomial counting procedure just described.

We now apply this formalism to one of the examples in Table 2. Consider the rank 4 example over $W C P_{1,1,1,1,2}^{4}$. From $(4.7)$ we have $\operatorname{dim} H^{2}(E)=$ $\operatorname{dim} H^{2}\left(O_{D}(-2)\right)-\operatorname{dim} H^{2}\left(O_{D}^{\oplus 4}(3)\right)$. We can compute the dimension of $H^{2}\left(O_{D}(-2)\right)$ from the three exact sequences derived from (4.1),

$$
\begin{aligned}
& 0 \rightarrow O_{K}(-6) \rightarrow O_{K}(-2) \rightarrow O_{D}(-2) \rightarrow 0, \\
& 0 \rightarrow O_{W C P^{4}}(-8) \rightarrow O_{W C P^{4}}(-2) \rightarrow O_{K}(-2) \rightarrow 0, \\
& 0 \rightarrow O_{W C P^{4}}(-12) \rightarrow O_{W C P^{4}}(-6) \rightarrow O_{K}(-6) \rightarrow 0 .
\end{aligned}
$$

Using these sequences and the discussion in the last paragraph on computing the dimension of line bundle cohomology over weighted projective spaces, it is straightforward to see that $\operatorname{dim} H^{2}\left(O_{D}(-2)\right)=118$. Similar analysis shows that $\operatorname{dim} H^{2}\left(O_{D}(3)\right)=4$. Thus, $\operatorname{dim} H^{2}(E)=102$, in agreement with our earlier computation of the third Chern number of this example. Again, this provides a nice check on our results and also, hopefully, conveys the ease with which the topological properties of these $(2,0)$ examples can be computed. In fact, the ability to express the cohomology of these examples by monomial representatives gives us further information on the discrete symmetry transformation properties of the fields in our theory. This, as is familiar from $(2,2)$ model building, serves as a powerful tool in the construction of the low energy Lagrangian. Although we shall not do so here, one can presumably generalize the monomial based Yukawa coupling algorithm [48] to the present situation. Thus, the low energy models following from these $(2,0)$ compactifications can be built with essentially the same techniques employed in the more familiar $(2,2)$ context. $^{10}$

10 The computation of the full Higgs spectrum, however, requires calculating $H^{*}\left(\Lambda^{2}(E)\right)[14,17,36]$. Unfortunately, a method for doing this has not as yet been worked out 
Furthermore, as in the $(2,2)$ case, there are some powerful nonrenormalization theorems which greatly enhance the applicability of tree level Yukawa coupling computations. Recall that in [19] we gave two proofs that the $27^{3}$ coupling are given by the lowest order computation: one made use of the instanton approximation to the sigma model while the other went beyond this approximation by making use of the $(0,2)$ superconformal Ward identities. For $(2,0)$ models we are limited to the first technique; nonetheless some strong statements can be made with little effort as the generation of superpotential corrections essentially comes down to whether fermi zero modes in an instanton background can be absorbed by the correlator of interest. In the $(2,2)$ case, for example, a three point function involving $\overline{27}$ modes can absorb the four $\lambda$ zero modes in an instanton background [16] while a correlator involving three 27's cannot [19]. This is one way of seeing that the latter couplings are not corrected. For the $(2,0)$ theories constructed with a rank $r$ bundle as above, we have 2(r-1) [17] $\lambda$ zero modes in an instanton background. For $r=4$ this implies that of the renormalizable couplings only the $\phi^{3}$ coupling, with $\phi \in H^{1}($ EndV) can receive instanton corrections. For $r=5$ (that is, the $S U(5)$ examples), we have eight $\lambda$ zero modes which cannot all be absorbed by any of the renormalizable interactions. Thus, the full renormalizable superpotential in such models is not renormalized by world sheet instantons and is thus given by its tree level value. ${ }^{11}$ Hence, the important phenomenological couplings in these relatively complicated geometrical vacua are given exactly by lowest order computations.

\section{Conclusions}

We have discussed some aspects of superconformal string compactifications which arise as orbifolds of nonminimal model Landau-Ginzburg theories. By using the path integral argument of [9] and by following a simple formalism rooted in elementary singularity theory, we have seen that an abundance of new Calabi-Yau manifolds can be constructed. This class of manifolds offers an important generalization of the well studied complete intersections as they allow for the incorporation of Calabi-Yau orbifolds with their associated singularities. In fact, this class is sufficiently general as to stand a chance of exhausting all possible Calabi-Yau constructions. We have emphasized one interesting application of these vacuum manifolds - the construction of numerous stable string vacua with only $(2,0)$ world sheet supersymmetry. These examples are the first which are not free theories and which are not simply deformations of underlying $(2,2)$ theories. It thus appears that this more general sector of the space of supersymmetric string vacua, in contrast to prior expectations, is quite extensive.

From the point of view of phenomenology, none of the examples we have constructed have a realistic number of generations, so, as yet, there is no point in detailed model building. As we have noted, though, the nontriviality of the hidden sector gauge bundle provides a nice tool for constructing models with fewer

11 We are assuming, of course, the complete validity of the instanton expansion 
generations. Furthermore, as shown in [17], the standard $(2,2)$ procedure of reducing the number of generations by passing to a quotient of the original theory by some discrete group action applies as well in $(2,0)$ theories, so long as the action lifts to the gauge bundle. In [17], for example, it was shown that the 100 generation $S O(10)$ theory can be modded out by a $Z_{5}$ symmetry, thus giving rise to a 20 generation example. It would be interesting to see if there are suitable symmetries for the examples presented here. Besides reducing the number of generations, modding through by such an action would allow the flux mechanism to operate [45] and hence aid breaking these unifying groups to the standard model. We have also seen that the extraction of the data necessary for detailed model building in these $(2,0)$ models is, to a large extent, no more difficult than in $(2,2)$ theories. In particular, we have shown that for the examples with an $S U(5)$ unifying group, tree level calculations of renormalizable superpotential terms are, in fact, exact.

We have thus seen that these relatively complicated geometrical constructions give rise to physical properties which, by the above, are simple to calculate exactly. This is the same situation which was realized some time ago for the $(2,2)$ Calabi-Yau constructions. It seems likely that, as in the $(2,2)$ case, this ability to extract couplings exactly through lowest order computations indicates that these theories will ultimately have a very simple and natural description as conformal field theories.

Acknowledgements. We would like to thank J. Distler, J. Harris, S. Roan, C. Vafa, N. Warner and S-T. Yau for interesting and useful discussions and P. Miron for bringing reference [22] to our attention. This work is supported by Department of Energy contract no. DE-FG02-88ER25065.

Note added in proof. Since completing this work, we have learned that Candelas, Lynker and Schimmrigk [49] have vigorously continued the construction of Calabi-Yau manifolds along the lines we have outlined in Sect. 2 and have found some striking results. These include a surprising symmetry in the distribution of Euler numbers about $\chi=0([50])$ as well as the construction of a number of three generation examples in weighted projective space.

\section{References}

1. Candelas, P., Horowitz, G., Strominger, A., Witten, E.: Nucl. Phys. B258, 46 (1985)

2. Dixon, L., Harvey, J., Vafa, C., Witten, E.: Nucl. Phys. B274, 285 (1986)

3. Kawai, H., Lewellen, D., Tye, S. -H. H.: Phys. Rev. Lett. 57, 1832(1986); Nucl. Phys. B288, 1 (1987)

4. Antoniadis, I., Bachas, C., Kounnas, C.: Nucl. Phys. B289, 87 (1987)

5. Narain, K. S., Sarmadi, M., Vafa, C.: Nucl. Phys. B288, 551 (1987)

6. Lerche, W., Lust, D., Schellekens, A. N.: Nucl. Phys. B287, 447 (1987)

7. Gepner, D.: Nucl. Phys. B296, 380 (1987)

8. Gepner, D.: Phys. Lett. 199B, 380 (1987)

9. Greene, B., Vafa, C., Warner, N. P.: Nucl. Phys. B324, 371 (1989)

10. Martinec, E.: Phys. Lett. 217B, 431 (1988) and in Knizhnik, V. G. Memorial Volume L. Brink, et al. (eds)

11. For a recent review, see L. E. Ibanez: Proceedings of the Maryland Strings '88 Workshop, World Scientific and references therein

12. Font, A., Ibáñez, L. E., Mondragon, M., Quevedo, F., Ross, G. G.: Phys. Lett. B227, 34 (1989); Font, A., Ibáñez, L. E., Quevedo, F., Sierra, A.: The construction of "Realistic" four-dimensional strings through orbifolds. CERN preprint CERN-TH-5326/89 
13. Greene, B., Plesser, M. R.: Harvard preprint HUTP-89/A043

14. Witten, E.: Nucl. Phys. B268, 79 (1986)

15. Dine, M., Seiberg, N.: Nucl. Phys. B306 137 (1988)

16. Dine, M., Seiberg, N., Wen, X. G., Witten, E.: Nucl. Phys. B278 769 (1987); B289, 319 (1987)

17. Distler, J., Greene, B.: Nucl. Phys. B304, 1 (1988)

18. Greene, B.: Phys. Rev. D (to appear)

19. Distler, J., Greene, B. R.: Nucl. Phys. B309, 295 (1988)

20. Yau, S. -T.: In: The Proceedings of the Argonne Symposium on Anomalies, Geometry and Topology, (1985)

21. Strominger, A., Witten, E.: Commun. Math. Phys. 101, 341 (1985)

22. Kim, J., Koh, I., Yoon, Y.: Phys. Rev. D33, 2893 (1986)

23. Vafa, C., Warner, N. P.: Phys. Lett. 218B, 51 (1989)

24. Greene, B., Roan, S. -S., Yau, S. -T.: in preparation

25. Vafa, C.: Harvard Preprint HUTP-89/A018

26. Roan, S. -S., Yau, S. -T.: University of San Diego preprint, 1985

27. Arnold, V. I., Gusein-Zade, S. M., Varchenko, A. N.: Singularities of differentiable maps. Vol. I. Basel, New York: Birkhäuser 1985

28. Yau, S. -T.: private communication

29. Kazama, Y., Suzuki, H.: Nucl. Phys. B321, 232 (1989)

30. Candelas, P., Lutken, C. A., Schimmrigk, R.: Nucl. Phys. B306, 113 (1988)

31. Aspinwall, P., Greene, B., Kirklin, K., Miron, P.: Nucl. Phys. B294, 193 (1987)

32. Green, P., Hubsch, T.: Texas preprint UTTG-4-88, 1988; Candelas, P., Green, P., Hubsch, T.: Texas preprint UTTG-10-8, 1988; Phys. Rev. Lett. 62, 1956 (1989)

33. Hull, C., Witten, E.: Phys. Lett. 160B, 398 (1985)

34. Boucher, W., Friedan, D., Kent, A.: Phys. Lett. 172B, 316 (1986)

35. Sen, A.: Nucl. Phys. B278, 289 (1986); Nucl. Phys. B284, 423 (1987)

36. Greene, B. R., Kirklin, K. H., Miron, P. J.: Nucl. Phys. B274, 574 (1986)

37. Cvetic, M.: Phys. Rev. Lett. 59, 2829 (1987)

38. Uhlenbeck, K., Yau, S. -T.: Commun. Pure and App. Math., Vol. XXXIX, 257 (1986)

39. Maruyama, M.: J. Math. Kyoto 18, 557 (1978)

40. Harris, J.: Duke Math. J. 46, 685 (1979)

41. Distler, J.: private communication

42. Griffiths, P., Harris, J.: Principles of Algebraic Geometry. New York: Wiley 1978

43. Distler, J., Greene, B.: unpublished

44. Okonek, C., Schneider, M., Spindler, H.: Vector bundles on complex projective spaces. Basel, New York: Birkhäuser 1980

45. Witten, E.: Nucl. Phys. B258, 75 (1985)

46. Hubsch, T.: Commun. Math. Phys. 108, 291 (1987); Green, P., Hubsch, T.: Commun. Math. Phys. 109, 99 (1987)

47. Dolgachev, I.: In: Group actions and vector fields. Lecture Notes in Mathematics Vol. 956. Berlin Heidelberg New York: Springer 1982

48. Candelas, P.: Nucl. Phys. B298, 458 (1988)

49. Candelas, P., Lynker, M., Schimmrigk, R.: University of Texas preprint, 1989

50. See also Greene, B., Plesser, M. R.: Nucl. Phys. B (to appear)

Communicated by S.-T. Yau

Received November 28, 1989 
